\title{
Correction to: Outcomes of plasma exchange for severe dilated cardiomyopathy in children
}

\author{
Keiichi Koizumi ${ }^{1} \cdot$ Minako Hoshiai $^{1} \cdot$ Takeshi Moriguchi $^{2} \cdot$ Takako Toda $^{1} \cdot$ Nobuyuki Katsumata $^{1} \cdot$ Hiroaki Kise $^{1}$. \\ Yohei Hasebe $^{1}$ - Yousuke Kouno ${ }^{1}$. Junko Goto ${ }^{2}$. Norikazu Harii ${ }^{2} \cdot$ kenichi Matsuda $^{2} \cdot$ Shigeaki Kaga $^{3} \cdot$ Shoji Suzuki $^{3}$. \\ Kanji Sugita ${ }^{1}$
}

Published online: 18 April 2018

(c) Springer Japan KK, part of Springer Nature 2018

\section{Correction to: Heart Vessels (2017) 32:61-67 https://doi.org/10.1007/s00380-016-0830-1}

In original publication of the article, some of the co-author's names were not included. The correct author group is published in this article.

In addition, the below reference was not included in the original publication of the article.

27. Moriguchi T, Koizumi K, Matsuda K, Harii N, Goto J, Harada D, Sugawara H, Hoshiai M, Kise H, Baba A (2017) Plasma exchange for the patients with dilated

The original article can be found online at https://doi.org/10.1007/ s00380-016-0830-1.

Keiichi Koizumi

kkeiichi@yamanashi.ac.jp

1 Department of Pediatrics, Faculty of Medicine, University of Yamanashi, 1110 Shimokato, Chuo, Yamanashi 409-3848, Japan

2 Department of Emergency and Critical Care Medicine, Faculty of Medicine, University of Yamanashi, Yamanashi, Japan

3 Department of Surgery, Faculty of Medicine, University of Yamanashi, Yamanashi, Japan cardiomyopathy in children is safe and effective in improving both cardiac function and daily activities. J Artif Organs 20:236-243

The reference should be cited in the second sentence under the section Plasma exchange.

Patients with severe heart failure were regarded as having hemodynamic instability. We performed slow PE plus continuous hemodiafiltration (SPE + CHDF), as described previously [14, 15, 27].

Figure 2 was published incorrectly in the original publication of the article. The correct Figure 2 is published in this article. 
(a) LVEF

(\%) 80

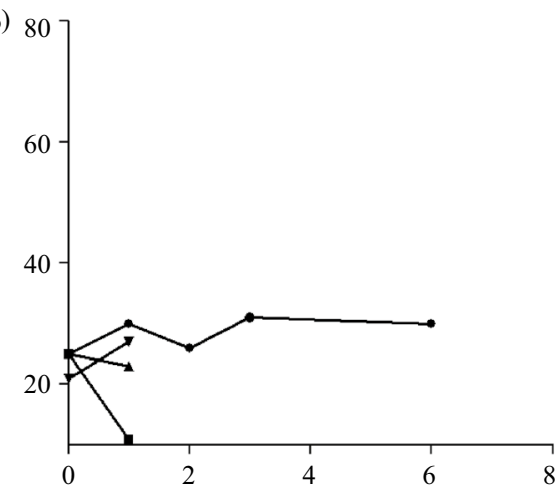

(b) BNP

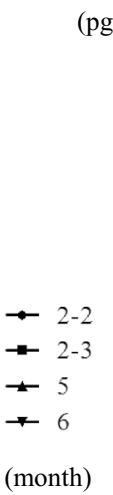

2500

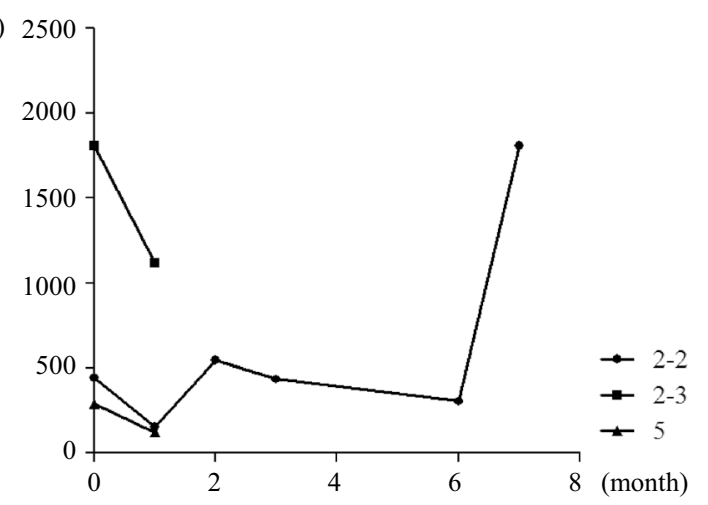

Fig. 2 Changes in LVEF and BNP after the repeated PE. a LVEF. b BNP. BNP plasma brain natriuretic peptide, $L V E F$ left ventricular ejectionfraction, and $P E$ plasma exchange 